\title{
Organizational networks with self-standing interests
}

\author{
Vasily Say ${ }^{1, *}$, Sergey Sizyi ${ }^{2}$, and Igor Gromov ${ }^{1}$ \\ ${ }^{1}$ Ural State University of Railway Transport, Department of railway track and railway construction, 620034 Yekaterinburg, \\ Russian Federation \\ ${ }^{2}$ Ural Federal University, Department of Algebra and Fundamental Informatics, 620083 Yekaterinburg, Russian Federation
}

\begin{abstract}
With the development of world economy gradual replacing of traditional organizational structures by organizational networks, take place. New organizational complexes set difficult tasks for researchers considering optimum building and functioning of organizational networks. In these conditions, a key focus of organization network research is simulating the interaction process as well as network's integrated quantitative assessment based on this simulation. A distinctive feature of the applied simulating approach is formalization of interaction processes between the integrator and network entities taking into account their contradictory interests. The integrated quantitative assessment of a network is offered proceeding from understanding that the general purpose of functioning is satisfying society needs with products and services of a network.
\end{abstract}

\section{Introduction}

Nowadays, the task of creating organizational networks is at the peak of its relevance for Russian economy. Such structures, based on associational or holding method, are being built now with consideration of the world's best experience. However, the organizational and economic field, which has developed after the country's changeover to a market economy, was not created in the course of evolution, but by means of strict administrative actions. Universally there was the process of restructuring in the form of downsizing. Thus, a process of radial-flow separation, which undoubtedly influenced the structure of organizational networks, had preceded the process of networking.

The authors of this article apply to the worldrenowned examples of companies' network creation and develop the theoretical aspects of the organizational network formation with consideration of regional economy mentality, since a well-organized process can be a key to a network's prosperity.

\section{Related literatures}

Formation, adjustment and operation of an organizational net-work depend on its type. Manuel Castells divided all the net-works into two groups, the first - a multi-directional network model, introduced into life by small and medium-sized enterprises, the second licensing and subcontracting production model under an «umbrella» corporation [1-3].

However, it is common to define a network organization as one that is fast and flexible in adapting to changes in the underlying environment [4-7]. Umbrella Corporation is that element of control of this stakeholder group holding the executive power should be a central topic for stakeholder theory [8-9].

In further studies, a network is considered as a formation around a large company, that is, «umbrella» corporation.

Under a network with self-standing interests, we understand the presence in an umbrella corporation and all networking companies their own, in most cases, conflicting interests. Thus, decentralization and increased flexibility of the structure may cause so called «articulation errors», defined by Guy Benveniste as «...the partial or total lack of fit between what is wanted and what is available» (cited from Castells 1996-1998, 178).

The problem of elimination of «articulation errors» in highly decentralized structures is complicated and often not solvable issue, and network adjustment in a constantly changing internal and external environment is a difficult continuous process.

M. Castells confirms our assumptions by considering it as a specific form of an enterprise, which assets are composed by the segments' intersection of autonomous goal systems.

However, further we will consume, that all the companies included in a network are connected by a common aim, and this is one of the key stages of organizational network formation. By intercommunity of the network aim, we suppose satisfying society's needs in products or services or «the production of useful things», as H. Ford claimed.

Our assertions resonate with [10] «Successful partnerships and collaborations in service of sustainable effectiveness will require individual organizations to change their objective function and build new and varied internal and external capabilities», in accordance with 
[11] «Every agent realizes his full potential and has no incentive to deviate from either staying independent or from the endogenously determined partner and payoff. The partnership formation problem contains the widely studied assignment market problem as a special case».

During the network modelling it is also needed to take into account the interests and priorities of its members. J. Tullberg in the work «Stakeholder theory: Some revisionist suggestions» maintains that the priority of stakeholders implies distancing from general altruistic philosophy that argues against, but not for, giving special consideration to the company's stakeholders [8].

The network structure is formed on the basis of integrator properties and the elements included in the network. Thus, the network is formed endogenously: «In equilibrium, the partner-ship formation and the payoff distribution are endogenously determined. Every agent realizes his full potential and has no incentive to deviate from either staying independent or from the endogenously determined partner and payoff» [11].

Another important factor in the formation of networks is that the company gets into tough operating environment. Fernando Vega-Redondo also confirms this fact in «Network organizations»: «The main conclusion obtained is that, as the environmental volatility increases, the optimal functioning mode of the organization sharply switches from being totally flexible to being completely rigid, i.e. no intermediate configurations are essentially ever optimal» [4].

\section{The model}

Formalization of large, sometimes hardly controllable social and economic systems requires a mandatory development of a graphical analytic representation of the processes. The organizational structure of a network, which reflects the main idea of business entities' interaction in the networks with self-standing interests, is presented in the form of the model in Fig. 1.

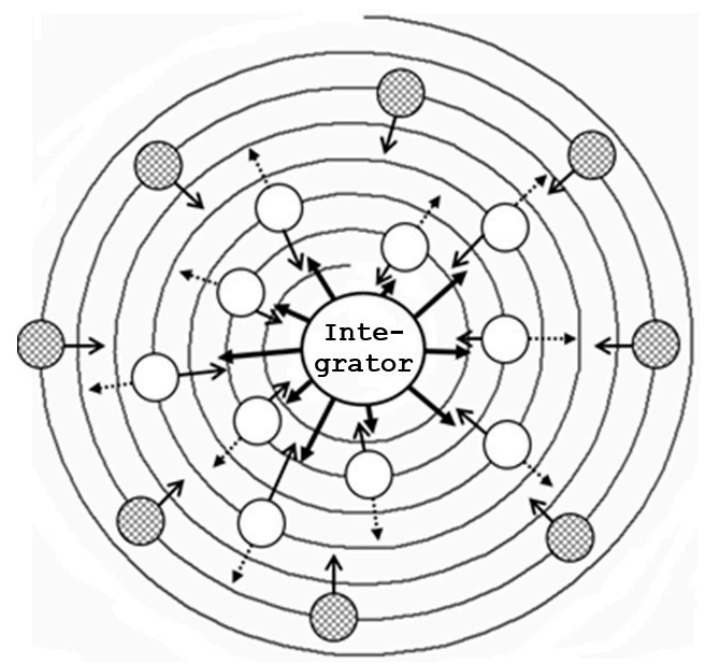

Fig. 1. Graphical analytic model of an organizational network with self-standing interests: $\bigcirc$ - network business entities; $\bigcirc-$ market business entities; $\longrightarrow$-attraction force of entity to a network; ……......
- attraction force of entity to a market; integrator interactive force to network and market entities.

The suggested model is a follow-up of radialplanetary type construction theory for organizational interaction in network structures [12]. A distinctive feature of the proposed modeling approach is formalization of the interaction between the integrator and network elements (business entities) in respect of their conflicting interests [13].

To formalize the relationship between integrator and net-work business entities let us present their interaction by functioning of attraction - repulsion forces [14]. We can draw a successful analogy between interaction of network entities and gravity interaction of material bodies within this approach. Functioning in common economic and legal space subjects inevitably gain certain connections and this phenomenon leads to appearing of attraction and repulsion economic forces.

So, for quantitative evaluation of «attraction forces» non-dimensional indicators accepting values in the interval $[0 ; 1]$ were introduced. We will consider their content representation [15]. Integrator tending to interact with a business entity $\left(K_{\text {int }}\right)$ can be calculated by a formula:

$$
K_{\text {int }}=1 /\left(1+\alpha_{1}\left(R-R_{a c c}\right)^{2}\right)+\alpha_{2} K_{\text {inf }}-\alpha_{3} P_{f r},
$$

where: $R$ - profitability of a business entity; $R_{a c c}-$ acceptable profitability of a business entity; $K_{\text {inf }}-$ indicator considering informal relationship between superiors; $P_{f r}-$ average percent of production fraud during a certain period; $\alpha_{1}, \alpha_{2}, \alpha_{3}$ - weight coefficients.

Attraction of a business entity to a network:

$$
K_{\text {att.n }}=1 /\left(1+\alpha_{4}\left(R-R_{\text {des }}\right)^{2}\right)+\alpha_{5} K_{\text {inf }}-\alpha_{6} K_{\text {sup }}
$$

where: $R_{\text {des }}$ - desirable profitability of a business entity; $K_{\text {sup }}$ - indicator characterizing integrator support of business entity; $\alpha_{4}, \alpha_{5}, \alpha_{6}-$ weight coefficients.

We can calculate $K_{\text {sup }}$ indicator by a formula:

$$
K_{\text {sup }}=\beta K_{a d v}+(1-\beta)\left(C_{e c} / N_{e c}\right),
$$

where: $K_{a d v}$ - indicator accepting value «1», if between the integrator and the subject exists the advance payment system, and « $\langle 0 »-$ if there is no advance payment system; $C_{e c}-$ economic opportunities regarding the network entity; $N_{e c}$ - economic needs of a business entity for the purpose of maintaining stable and efficient production activity; $\beta$ - weight coefficient.

Further, we will consider «business entity attraction towards market» indicator $-K_{\text {att.m }}$ :

$$
K_{\text {att.m }}=1 /\left(1+\alpha_{7}\left(R_{m}-R_{\text {des }}\right)^{2}\right)+\alpha_{8}(H H I / 5000-1),
$$

where: $R_{m}$ - business entity profitability in the case of its production capacities realization in the market; HHI Gerfindal - Girshmana's index for i-business entity branch; $\alpha_{7}, \alpha_{8}-$ weight coefficients.

The entered indicators play an essential role in investigation of organizational networks with selfstanding interests. Quantitative evaluation of network 
attraction forces allows projecting interaction stability of an integrator and a business entity in the conditions of dynamically changing relationship between them [16]. The study of the stability of organizational networks dedicated to a number of works, for example [17-19].

We will note that at research of a concrete organizational network the list of factors needs to be specified depending on research problems and specific nature of network production activity management.

The studied type of a network («umbrella» corporation) is characterized by existence of continuous administrative influence on business entities from the integrator. Administrative influence is understood as organizational, administrative and economic impact of the integrator on economic entities and a network in general.

Administrative influence has an integration character and is directed to a network strengthening. On the one hand, such influence lets the integrator realize its task solving strategy, and on the other hand, it provides for an economic entity effective work in a network.

\section{Quantitative network evaluations}

The quantitative evaluation of organizational networks with self-standing interests functioning is necessary for giving grounds for the options of their development. Elaboration of quantitative evaluation technique demands carrying out various industrial experiments directed to searching of optimum pro-portions of an organizational network functioning. The lack of opportunity to make the necessary organizational experiments in practice sets for a researcher the task of development a simulating model of organizational network, which allows presenting all the possible options and schemes of business entities interaction in a network. This can also help to test all the administrative decisions by means of numerical experiments, to estimate and choose among them the most acceptable from the point of view, both a separate economic entity, and system in general.

Thus, under organizational network quantitative evaluation we will understand some integrating indicator $K_{\text {ind }}$, which estimates organizational technical and economic level of its development.

We will consider the main idea of $K_{\text {ind }}$ indicator formation. As the purpose of organizational network functioning is to satisfy the needs of society in products or services, it will be logical to estimate the activity of a network by the following relation:

$$
K_{\text {ind }}=\left(Q_{p r}-R_{e x}\right) / W_{p},
$$

where: $Q_{p r}$ - the projected production volume (volume of services), which was defined taking into account technical and economic level of entities' development and the quality of their interaction; $R_{e x}-$ external risks for network functioning; $W_{p r}$ - production capacities of a network.

$Q_{p r}$ is mathematical expectation function of production and economic network activity results, calculated by a set of tests. Testing of an organizational network assumes imitation (generation) of various organizational and production situations for integrator and network entities. Mathematical formalization of $Q_{p r}$ proposed in [20].

Finding $R_{e x}$ is a challenging research task. This can be explained by the fact that casual, hard to project variables, describes the moment of negative external attack on a network, its stability and consequences. The choice of mathematical apparatus for statistical modeling and probability theory can become a rational way of this indicator formalization.

As the generated production situations have various probabilities of happening, the values of $Q_{p r}$, received during the trials, are not equiprobable. Obviously, various negative consequences from external influence are not equiprobable. Then, the nature of dependences of $Q_{p r}$ and $R_{e x}$ on production situation happening probability $P_{\text {sit }}$ can be presented in one system of coordinates (Fig. 2).

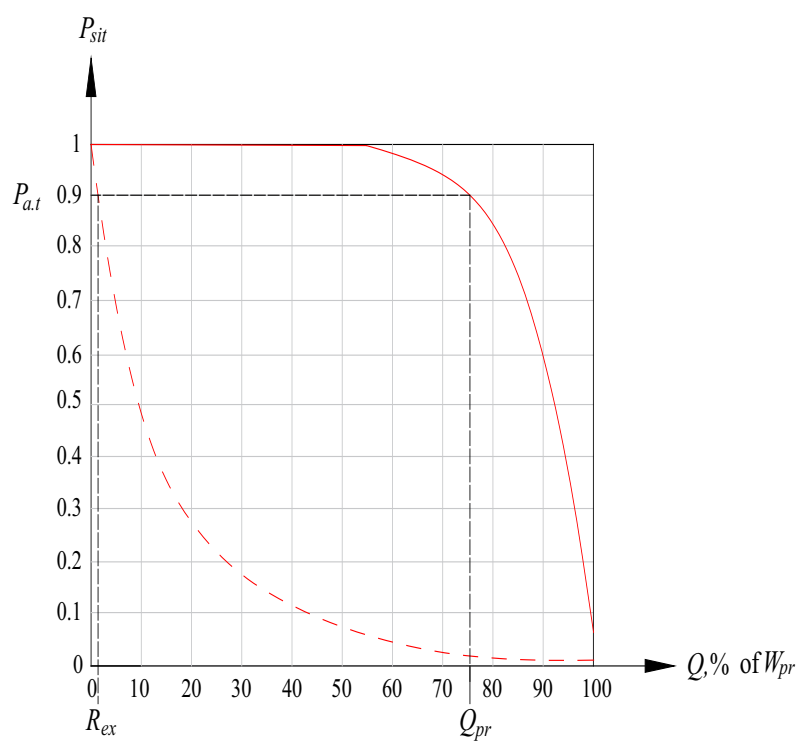

Fig. 2. Results of organizational network activity and probability of their obtaining: $P_{a . t}-$ probability of almost true event.

It is rational to predict the results of production and economic organizational network activity with probability of almost reliable event. Considering the practice of carrying out statistical tests in other areas, we will accept $P_{a . t}=90-95 \%$. Such probability provides the acceptable accuracy of the forecast and the width of production economic network activity results interval $\left[Q_{p r} ; W_{p r}\right]$.

Practical importance of the presented dependences is that they simplify the process of organizational network adjustment and allow planning its functioning risks.

\section{Organizational network adjustments}

The obligatory condition of ensuring the effective functioning of network structure in commodity and service market - is its continuous adjusting based on the use of quantitative network evaluation. 
We will note that the formalized selection of economic entities from a free market is a guarantee of stability and efficiency of an organizational network. Integrator expects from all the network members positive activity results, increasing a network commercial sufficiency, its sustainability, and functional reliability. It is obvious that business entity network entering is mostly effective if $\Delta K_{\text {ind }} \rightarrow \max$. That is, with the choice of the entity from a set of possible options, the integrator aims at boosting the integrated network index.

A possibility of organizational network adjustment using the offered quantitative evaluation is demonstrated through adjustment results of a production organizational network with poor effectiveness. In the studied network, external functioning risks $\left(R_{e x}\right)$ are relatively low and were not considered in the course of quantitative evaluation. The main results of adjustment are given in Fig. 3.

It is obvious that logical actions of network adjustment should lead to integrated index increasing. At the initial condition of the network, an integrated index was 0,31 , and after four adjustment stages the index reached 0,64 . The most popular business administration decisions were tested at different stages of adjustment: change of integrator financial policy towards better satisfaction of network members' interests; increasing network stability due to addition of a new network member; strengthening of network support to the level of network members' needs; reservation of entities' production capacities.

The received results confirm that the quantitative evaluation adequately reflects the change of key network parameters and can be used for further research of organizational networks.

Initial condition of the network: $K_{\text {ind }}=0,31$.

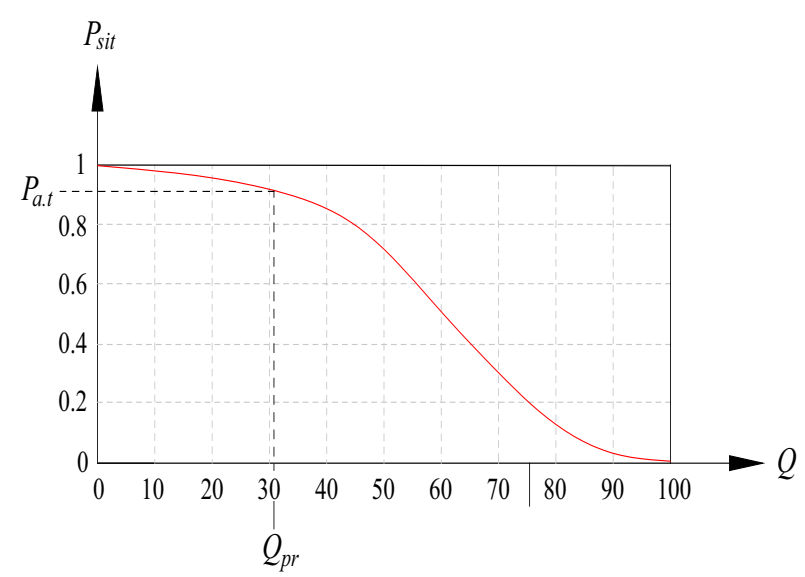

Change of integrator financial policy towards better satisfaction of network members' interests: $K_{\text {ind }}=0,46$.

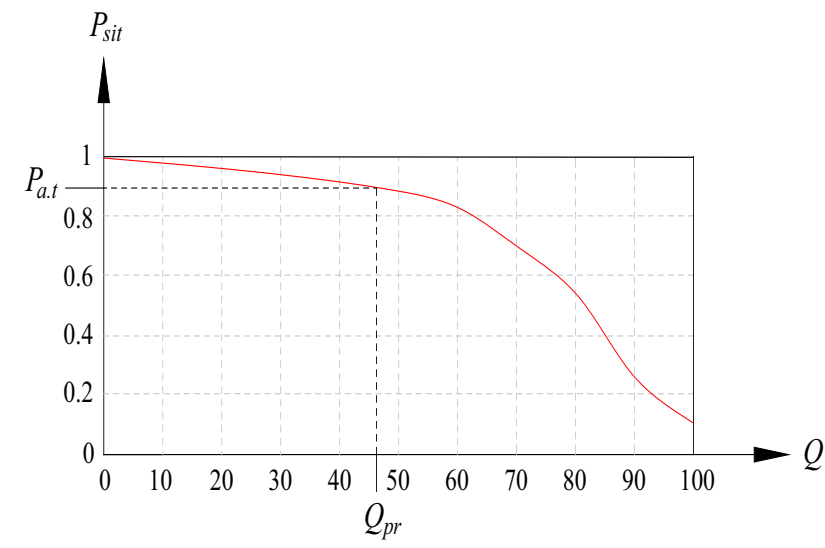

Formation of production capacities reserves due to inclusion in a net-work a new member: $K_{\text {ind }}=0,54$.

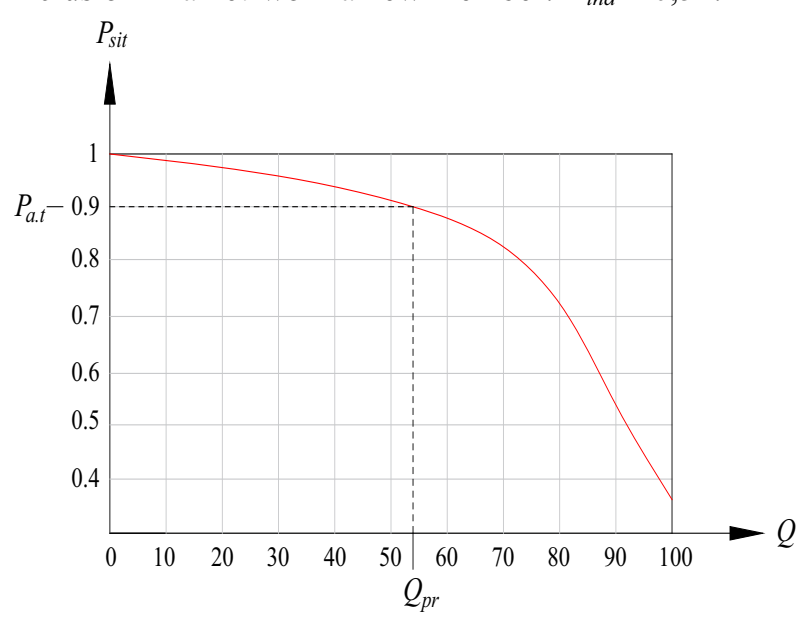

Strengthening of network support to the level of network members' needs: $K_{\text {ind }}=0,60$.

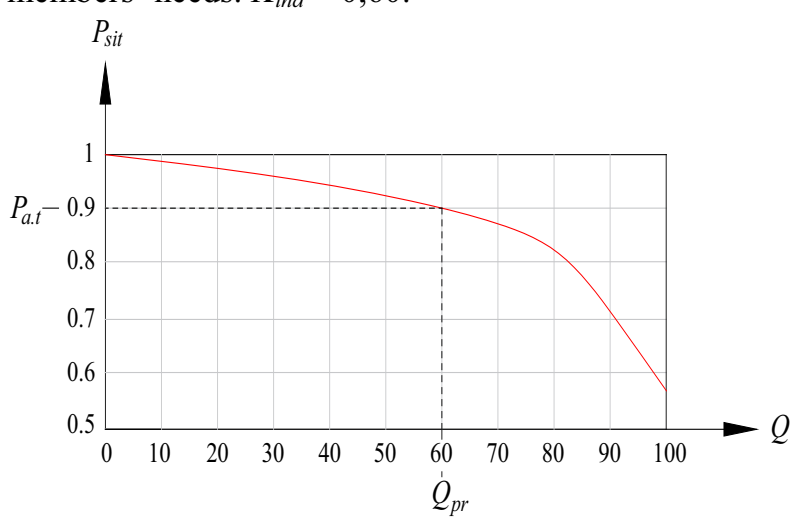

Reservation of $10 \%$ finished goods for the next period: $K_{\text {ind }}=0,64$. 


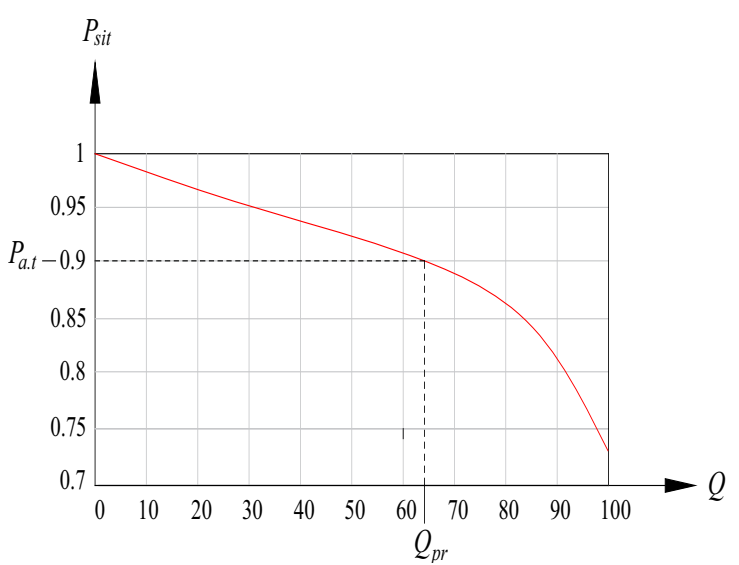

Fig. 3. Consecutive assessments of low effective organizational network's adjustment stages.

\section{Summary and conclusion}

So, in the offered graphical analytic model the main ideas of the theory of legal entities' interaction simulating in organizational network with self-standing interests are presented. Indicators of integrator and network entities' attraction are formalized in the form of functions. The functions' variables are degrees of satisfaction the corresponding interests. Proceeding from a need of each network, to revise the list of the evaluated interests, linear estimated forms were chosen as the most rational form of convolution.

The suggested approach of a network quantitative evaluation makes it possible to objectively analyze the production and economic activity of the whole network structure, network integrator and included economic entities, to estimate a network interaction, to project the necessary network parameters and to plan a network development.

The main advantages of suggested theoretical ideas and model representations are universality, convenience of computer realization, possibility of stage-by-stage calculation and adjustment of network organizational structures.

Further researches are aimed at development of complex simulating model of organizational network with self-standing interests. This will allow: to generate and quantitatively estimate various production scenarios; to investigate the influence of different load-level and capacity schemes of economic entities on indicators of the whole organizational network; to prove boundary values of production and economic activity of enterprises, which form a network and influence its durability and sustainability; to estimate the risks influence on interaction process of organizational network members.

\section{References}

1. M. Castells, The Rise of the Network Society. The Information Age: Economy, Society and Culture (Malden, MA, Oxford, 2010)

2. M. Castells, The Power of Identity. The Information Age: Economy, Society and Culture, 145-168 (2010)

3. M. Castells, End of Millennium. The Information Age: Economy, Society and Culture, 3, 377-378 (2010)

4. Fernando Vega-Redondo, J. of Complex Networks, 1, 72-82 (2013)

5. M. Van Alstyne, Forthcoming in j. of organizational computing, 7, 3 (1997)

6. J. Galbraith, J. of Org. Design, 2, 1-13 (2012)

7. Robert L. Cross, Salvatore Parise, Leigh M. Weiss, McKinsey Quarterly, 39, 301-323 (2003)

8. J. Tullberg, J. Behavioral and Experimental Ec., 42, 127-135 (2013)

9. Friedman, L. Andrew, Miles, Samantha, J. of Management Studies, 39, 1-21 (2002)

10. Philip H. Mirvis, Christopher G. Worley, Building Networks and Partnerships Emerald Group Publishing Ltd., 3, 1-34 (2013)

11. D. Talman, Zaifu Yang, J. of Math. Economics, 47, 206-212 (2011)

12. V. Say, S. Sizyi, Formation, operation and breakup of organizational network (USURT, Yekaterinburg, 2011)

13. V. Say, I. Gromov, J. modern problems of Russian transport complex, 3, 199-208 (2013)

14. I. Gromov, Yu. Moskvina, J. herald of USURT, 21, 34-40 (2014)

15. V. Antropov, I. Gromov, J. herald of USURT, 25, 29-37 (2015)

16. I. Gromov, J. herald of USURT, 58, 80-89 (2015)

17. Edward E. Lawler III, Christopher G. Worley, J. of Org. Design, 41, 265-270 (2012)

18. S. Trajanovski, J. Martin Hernandez, W. Winterbach, J. of Complex Networks, 1, 44-62 (2013)

19. L. Välikangas, G. Romme, J. of Org. Design, 2, 4453 (2013)

20. V. Say, I. Gromov, J. transport of the Urals, 46, 3-10 (2015 